\title{
"Al mensajero matara". La suplantación del autor por el mediador y tres textos bíblicos
}

\author{
Juan José Calvo García de Leonardo \\ Universitat de València (UVEG) \\ calvojj@uv.es
}

Resumen: La tendencia del traductor a intervenir en la transmisión de su original viene de antiguo. En el presente artículo, tras una introducción histórica y metodológica, se ilustrará esta postura con la exposición, análisis y discusión de las versiones de tres extractos del libro más traducido y retraducido del mundo: la Biblia. Desde el punto de vista de la tipología textual tenemos tres: uno informativo (el Prólogo al Evangelio de Juan), uno expresivo (el Cantar de los Cantares) y uno persuasivo o, como prefería llamarlo Nida, imperativo (el Padrenuestro). Partimos, básicamente, de la versión latina Vulgata como texto original y las versiones a distintas lenguas occidentales y en diversos momentos históricos, como textos meta.

Palabras clave: historia de la traducción; metodología traductológica; traducción cultural; substitución; coautoría.

\section{"Al mensajero matara". The mediator impersonates the author and three biblical texts}

Abstract: The tendency of the translator to participate in the transmission of his original is an ancient one. In the present paper, after a historical and methodological introduction, this stance will be illustrated by means of the exposition, analysis and discussion of the versions of three extracts from the most translated and retranslated book in the world: the Bible. From the point of view of text-typology, we have three: an informative (the Prologue to Saint John's Gospel), an expressive (The Song of Songs) and a persuasive or, as Nida would have it, an imperative one (The Lord's Prayer). We basically rely upon the Latin Vulgate as source text and the version into several Western European languages, at different historical periods, as target texts.

Keywords: history of translation; translationmethodology; cultural translation; substitution; co-authorship.

》 Calvo García de Leonardo, Juan José. 2016. “ “Al mensajero matara'. La suplantación del autor por el mediador y tres textos bíblicos”. Quaderns de Filologia: Estudis Lingüistics XXI: 57-71. doi: 10.7203/qfilologia.21.9312 

Paseábase el rey moro
por la ciudad de Granada;
cartas le fueron venidas
como Alhama era ganada;
las cartas echó en el fuego
y al mensajero matara.

Romance del rey moro que perdió Alhama (aprox. 1500)

\section{Presentación}

Cuando una musicóloga se mete a historiadora y llama archiduque a un Savoia, la obligación ética del traductor es advertirle del error; pero, si fuere pertinaz, la obligación contractual del traductor es verter el título espurio -insertando, como mucho y por prurito, un (sic). Muy por el contrario, si Shakespeare cree Bohemia litoral mediterráneo en The Winter's Tale y confunde Milán con Génova en The Tempest, o si Dante cree la Tierra inmóvil y central al Universo...el traductor no tiene licencia para intervenir. Y si dicha participación del mediador es ilegítima en el caso de los poetas, de los creadores mortales, mayor culpa habría con la palabra del Creador por excelencia. Si el traductor se creyera Dios, habría de verse perseguido por su criatura, como Victor Frankenstein, hasta los confines de la Tierra. Para los pueblos del desierto no cabe duda: la tradición semita lo prohíbe expresamente y el Corán, al menos como presupuesto formal, no se puede trasvasar a otras lenguas. Pero la palabra de Dios, el $\lambda o ́ \gamma o \varsigma$ de San Juan, es $\kappa \alpha \theta$ o $\lambda$ «ós, universal; y ello ha dado pie a intervenciones sin número.

\section{Primera parte: algunos presupuestos teóricos}

\subsection{El TL1 como sujeto de explotación y reelaboración}

\subsubsection{La tradición pragmática y material romana}

Desde la batalla de Corinto (146 a. C.) y la transformación de Grecia en provincia romana, junto con el territorio, la República se apropia de la cultura helenística. Un derecho sobre bienes comunes, publica materies (Horacio Ars Poetica V, 131), un derecho de conquista, como dirá San Jerónimo en el símil de su Epistula LVII ad Pammachium De optimo genere interpretandi (1962: 493). El hombre libre tenía derecho de uso, de disfrute y de abuso sobre sus propiedades y Roma ejerció esa 
"propiedad plena". Hasta tal punto llegará la aculturación, la naturalización asimiladora, que la civilización romana geminará en grecorromana. Y así, con el Imperio, cabe alguna matización y el texto original, el TL1, ya no podrá ser subsumido, sino figurar en paralelo, como cuando Quintiliano (Institutionis oratoriae libri XII 5: 4-6) habla de aemulatio y de certamen, con la atlética intención de superar al competidor, al original, en destreza retórica y en belleza poética.

Este modelo de apropiación pervivirá en Europa, sobre todo en la Francia Moderna, hasta el siglo XVIII. El cambio de mentalidad comienza en ese siglo, junto con el desarrollo, en Inglaterra, del concepto legal de derecho de autor, a partir de 1709, de tal forma que, cuando se descubren las ruinas de Herculano (1738) y de Pompeya (1748) reinando Carlos VII de Nápoles (luego Carlos III de España), ya no es lícito, como lo fuera la Antigüedad en su conjunto y desde la Edad Media, utilizarlas como cantera. Llegados los prerrománticos, el original deja de ser un consumible orgánico para fosilizarse en tótem, como nos recordará Nietzsche en su Fröhliche Wissenschaft de 1882 (Störig 1969: 136) cuando se burla del antiquarischem Spürgeist de la traducción romántica burguesa.

\subsubsection{La justificación subjetiva e ideológica}

La segunda justificación de la manipulación del TL1 no deriva de la objetividad de la victoria sino de la subjetividad ideológica. Es la que permitió a Perrot d'Ablancourt transmutar en defensor de la monarquía absoluta a Tácito (Von Stackelberg: 47), sin admitir que fuera versión ni emulación alguna (los paraphrase e imitation de Dryden) para poder aprovechar el prestigio del historiador romano. Es el falseamiento que se le echó en cara al doblaje del Séptimo Sello (Bergman Det sjunde inseglet, 1957). Es lo que nadie objeta cuando se comulga con la causa, sea ésta anti-patriarcal, anti-colonialista, anti-racista, anti-sexista o cualquier otra antítesis socialmente integrada o apetecida.

\subsection{La fidelidad al TL1}

\subsubsection{La revolución romántica: el Naturgenie}

En la mitología griega, Iris, la mensajera alada de Zeus repite la dicción del Dios Supremo con meticulosa exactitud, incluyendo la prosodia y 
todos los niveles de estilo, de manera similar a la inmediatez de la interpretación simultánea, que dificulta el distanciamiento emocional. El intérprete es, en este primer estadio, el oráculo de la Divinidad como lo son los profetas paleo-testamentarios; a la vez también es su mensajero o reencarnación ${ }^{1}$, desde el chamanismo y la pitonisa de Delfos, asentada sobre el ombligo de la Tierra, hasta los mistagogos helénicos. Este carácter se encuentra explícito en Platón, para quien los dioses entran en comunicación con los hombres por tres vías: el poeta inspirado (Ion), los mensajeros "cualificados" (Crátilo) y la posesión divina o el "entusiasmo" (Timeo). En un contexto cultural distinto, cuando se aparecen los tres viri (los ángeles o "mensajeros") ante Abraham (Gen. 18: 2-3), el patriarca se dirige a ellos con el singular: Domine. A nivel literario, en el Proemio a sus Annales, Ennio afirma habérsele aparecido Homero para asegurarle, profetizando al modo pitagórico, que su alma, al cabo de sucesivas metempsicosis, se había reencarnado en él ${ }^{2}$. Y esa es la revolución romántica: el autor es un Naturgenie ("genio natural/de la Naturaleza") como dice el Sturm und Drang y como a creador se le debe consideración, fidelidad y latría, por derecho natural.

\subsubsection{La traducción del texto sagrado: del hierolecto}

La Biblia se ha venido traduciendo desde la versión griega de los Setenta: una versión en todo punto coincidente, puesto que la palabra de Dios es inalterable (cf. Delisle \& Woodsworth, 1995: 162-164). Y aún así. "The Megillath Taanith (Roll of Fasting), which is assigned to the first century A.D., records the belief that three days of utter darkness fell on the world when the Law was translated into Greek" (George Steiner, 1975: 239). Joseph ben Matthias, conocido por los romanos como Flavio Josefo, afirma que Moisés era el traductor de Dios (ibidem), sustentando lo declarado por Filón de Alejandría de que la Biblia no es sino una traducción de la palabra de Dios (Kloepfer, 1967: 18). En consecuencia, si el $\lambda$ ó $o \varsigma$ divino crea el Universo, metamorfosear ese $\lambda$ ó $\gamma$ os conlleva el caos. Alterar en modo alguno la palabra de Dios es blasfemia y dogma que ha resultado utilísimo para uniformar la lengua y el pensamiento (al evitar la inseguridad y la atomización de la exége-

\footnotetext{
${ }^{1}$ Por eso el rey moro del romance mata al mensajero.

${ }^{2}$ T. R. Steiner (1975: 10) menciona a Chapman (The Tears of Peace, 1609) como caso análogo.
} 
sis) por toda la faja del Islam (Delisle \& Woodsworth, ibidem: 177s.). En un contexto paralelo, San Pablo recordará que no le es lícito al hombre proferir las palabras inefables oídas en el cielo: [...] in paradisum et audiuit arcana uerba, quae no licet homini loqui (2Co, 12: 4).

\section{Segunda parte: la traducción de la Biblia, de un TL1 globalmente persuasivo}

\subsection{La estrategia traductora como subsidiaria de la tipología textual}

Según Reiss (1971: 34-49), el tipo de texto presupone y rige la estrategia traductora: un texto "informativo" exige fidelidad semántica, uno "expresivo" permite recreación por analogía formal, uno "persuasivo" (apellbetont) abre las puertas a la versión, como dirá años más tarde (Reiss \& Vermeer, 1996), para ajustarse al skópos: al público y a la cultura receptores. Según dichas premisas, el texto que nos exigiría mayor fidelidad al autor es el Prólogo de San Juan y el Padre Nuestro el que más se adecuaría a la parroquia.

\subsection{El corpus y su análisis traductológico plurilingüe}

\subsubsection{El texto informativo: Juan 1}

Cuando Faust se decide a emprender la traducción de la Biblia al alemán (VI, Studierzimmer) no es que se enrede, como Lutero y sus colaboradores, durante semanas, en un "translema" (Roganova, 1971, en Vidal Claramonte, 1995: 30 s.; Santoyo, 1986), sino que se le atraganta

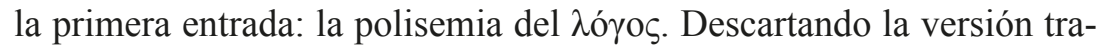
dicional (Wort), Faust roza una opción factible (Sinn) y termina por asumir sus condicionantes ontológicos y antonomásicos, como el individuo proto-romántico (Kraft) y de acción (Tat) que es.

San Juan prologa el único evangelio no sinóptico con una no-Teogonía, con su Genesis particular, sirviéndose para ello del nombre griego que fundamenta nuestra profesión: el $\lambda o ́ \gamma o s$. Aceptado como exégesis válida, el uerbum de la Vulgata lo siguen casi todos: verbo, palabra, parole, parola, Wort, word. Todos, excepto TLA ${ }^{3}$ que personifica, subordi-

\footnotetext{
${ }^{3}$ Casi todas las referencias en línea, generalmente dadas en abreviatura, están tomadas de https://www.biblegateway.com/. La versión primera de la Vulgata de San Jerónimo
} 


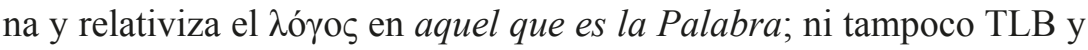
LBG, en versiones calcadas, que lo substituyen por la Segunda Persona (Before anything else existed, there was Christ, with God; Prima che esistesse qualsiasi altra cosa, c'era Cristo. Egli era con Dio) e incidir en el segundo problema de nuestro extracto.

Efectivamente y como veremos en el texto "expresivo", la dificultad mayor no reside en los translemas léxicos sino en los gramaticales. San Juan dice $\pi \rho$ òs tòv $\theta \varepsilon$ óv y San Jerónimo apud Deum. La preposición, que i.a. puede verterse como el commodi o de interés "a favor de", los espaciales y locativos "hacia", "junto a" y "cabe" o el comitativo "con", aporta, ella sola, suficientes haces de leña, porque fundamenta, o no, la consubstancialidad de la Segunda Persona de la Trinidad. Los alemanes, como de costumbre, siguen la Vulgata con el espacial bei, los franceses prefieren el comitativo avec, al igual que los españoles con. Los italianos, equilibradamente, ambos: CEI y LND presso, NR y LBG con. Las versiones en inglés suelen prefererir el comitativo, excepto W que prefirió el espacial at, WE que especifica were together ("estaban juntos") o WET in fellowship with ("en compañía de"). Los no trinitarios añaden un artículo para romper el dogma, como ya hiciera Arrio el bereber ${ }^{4}$.

se ha consultado en http://www.hs-augsburg.de/ harsch/Chronologia/Lspost04/Hieronymus/hie_v000.html

${ }^{4}$ Tenemos, entre otros muchos, lo siguientes:

a) a (1808, Thomas Belsham, The New Testament; 1822, A. Kneeland, The New Testament in Greek and English; 1829, J.S. Thompson, The Monotessaron; or, the Gospel History According to the Four Evangelists; 1863, Herman Heinfetter (pseudónimo de Frederick Parker), A Literal Translation of the New Testament; 1864, Benjamin Wilson, The Emphatic Diaglott; 1885, R. Young, Concise Commentary of the Holy Bible; 1911, G.W. Horner, The Coptic version of the N.T.; 1958, J.L. Tomanec, The New Testament of Our Lord and Saviour Jesus Anointed).

b) ein (1879, J. Becker, Das Evangelium nach Johannes; 1975, Sigfried Schulz, Das Evangelium nach Johannes).

Otros cambian nombre por modificador con casi el mismo efecto, al subordinarlo a Dios:

a) divine (1935, John M.P. Smith y Edgar J. Goodspeed, The Bible - An American Translation; 1955, Hugh J. Schonfield, The Authentic New Testament).

b) göttlich (1978, Das Evangelium nach Johannes de Johannes Schneider).

Este listado y otras referencias similares, en https://en.wikipedia.org/wiki/John_1:1 y http://simplebibletruths.net/70-John-1-1-Truths.htm [Acceso 2-01-2016]. 


\subsubsection{El texto expresivo: El Cantar de los Cantares 1: 5-6}

Para el segundo tipo de texto, el "expresivo", se ha elegido el gran canto amoroso del Antiguo Testamento: el Cantar de los Cantares. No se pretende analizarlo en su totalidad, ni siquiera en una parte substancial. Desde luego no embutiremos dos volúmenes (1993 y 2000) por apoyar un shibolleth, como se hiciera en la serie The Feminist Companion to the Bible compilada, entre otras, por Athalya Brenner ${ }^{5}$. Sólo analizaremos un color y una conjunción, que puede ser adversativa, concesiva o copulativa.

La primera discrepancia se centra en el color de la piel por su valor

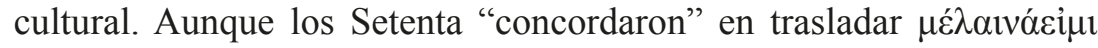

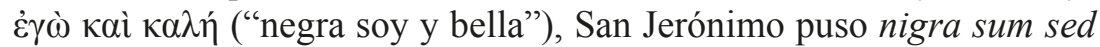
formonsa ("negra soy pero hermosa"). Abundando en la teoría del origen estepario del indoeuropeo, el latín distinguía entre el niger brillante y el ater mate; igual que el inglés antiguo tenía blaec o blac (> black) y sweart (cf. swarthy "cetrino"), tal y como lo atestiguan sus glosarios del latín ${ }^{6}$. Analicemos, pues, el color. Si suponemos la sulamita negra, lo más probable es que sea etíope ${ }^{7}$ : brillante como la joya del Ethiop's ear (RJ, I, v, 50), que le aparece Juliet ante Romeo, hermosa como la Aida verdiana. Y negra debe ser si ella misma se compara con las tiendas de Cedar y las cortinas del palacio de Salomón. Culturalmente hablando, la belleza es interior, no exterior; y tanto en la interpretación ortodoxa hebrea de la relación de Israel y Jahvé o en la de la Iglesia se puede asociar con "la piedra que desecharon los que edificaban vino a ser piedra angular". Salvando esto, la Septuaginta y la Vulgata traducen adecuadamente. Veamos las otras versiones.

L y S dan schwarz. NEG y LSG noire y LND nera. W, G, AV, ASV, NABRE y AMPC también optan por black. Por el contrario, todas las versiones españolas consultadas (P-TA y N-C, R-V, TLA, NTV y DHH)

\footnotetext{
${ }^{5}$ El segundo lo coeditará Carole Fontaine. Cf. además https://en.wikipedia.org/wiki/ Song_of_Songs. Ver, por otra parte, lo que se imagina Moya (2010: 166) sobre una traducción ideologizada del Cantar de los Cantares.

${ }^{6}$ Las citas se pueden consultar en el diccionario de Bosworth \& Toller en línea: http:// www.ling.upenn.edu/ kurisuto/germanic/oe_bosworthtoller_about.html

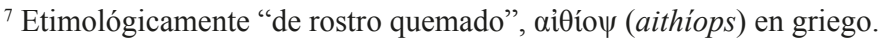

${ }^{8}$ Aunque con matices entre el Antiguo y el Nuevo Testamento, ver: Salmos 118: 22; Mateo 21: 42; Marcos 12: 10; Lucas 20: 17; Hechos 4: 11 y I Pedro 2: 7.
} 
preferirán morena, incluidas las poéticas de Fray Luis de León y Alonso Schökel,. BDS prefiere bronzée, la razón inmediata, obvia, es que el sol le ha tostado la piel. CEI escoge bruna y NR la difumina en scura. CEV y GNT se inclinan por dark y AMP glosa deeply tanned.

¿Qué escoger? Por mucho que prefiramos negra a morena, obscura, tostada (por el sol)/bronceada y demás tonalidades (¡que no sombras!) cromáticas del translema hebreo, el problema no reside ahí. La Sep-

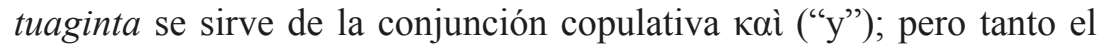
símil de las tiendas y las cortinas como las objeciones que a su belleza podrían elevar las otras muchachas, aquellas hijas de Jerusalén de la cultura receptora y dominante, contradicen la conjunción copulativa. Por el contrario, el sed ("pero") de la Vulgata es culturalmente lógico y poéticamente coherente. Y ahí está la pega, there's the rub como razonaba el príncipe Hamlet (II, i, 65). Aunque no aleguemos la traducción de la versión griega como TL1 ni la polisemia del adjetivo original, la censura imperante en las últimas décadas no puede aceptar la adversativa. Ontológicamente, hoy, una negra no puede no ser hermosa: es una contradictio in terminis, un oxímoron. Pero, repasemos las versiones. Las alemanas siguen la Vulgata con aber, la NEG y LSG dan mais y las italianas $m a$. También Fray Luis, P-TA, N-C y DHH con pero y R-V con mas. De entre las inglesas, W, G, AV, ASV, GNT, AMP y AMPC prefieren but. BDS se queda a medias, con la concesiva et pourtant. Finalmente, Schökel prefiere la copulativa. También TLA, que, además, hace hincapié en el hecho: Yo soy morena, sí, (...) Y también soy hermosa. CEV y NABRE elegirán igualmente and.

\subsubsection{El texto persuasivo: El Padrenuestro}

Llegamos al último texto, al "persuasivo". En el rito que fijaría la celebración de la Misa a partir del Concilio de Trento y la bula Quo primum tempore de San Pio V (1570) que, con mínimas correcciones, perviviría hasta el Concilio Vaticano II y el Novus ordo missae de Pablo VI (1969), dentro del carácter tetralingüe (latín, griego, hebreo y lengua vernácula) que lo caracterizaba, el Padrenuestro era uno de los componentes en lengua vulgar, pues su rezo corresponde a la comunidad de oficiante(s) y pueblo. Es el texto más conocido e, históricamente, el más trastocado de nuestro corpus. Por falta de espacio, nos centraremos en cuatro translemas de la versión de San Mateo (6: 9-13): 


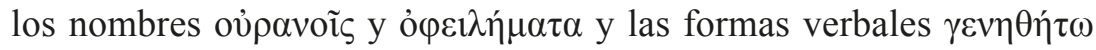

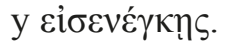

El primero, oủpavoĩs (ouranois), la pluralidad premoderna de los cielos superpuestos, refleja la ciencia coetánea, como el caelis de la Vulgata. Cierto, más adelante hay una reducción al singular caelo, pero no hacía falta nivelarlos, como sí lo hacen la versión actual española y las traducciones alemanas (Himmel). Ni R-V ni los valdenses lo vieron necesario. Las versiones en inglés antiguo, la anglia nortúmbrica de los Evangelios de Lindisfarne (LG) y la sajona occidental (A), usan el dativo plural heof(o)num y la conservadora, kéntica (C2), del inglés medio sigue con heuenes. Sorprendentemente, $\mathrm{C} 1$ ya usaba el dativo singular heofene y le seguirán, en el inglés medio tardío, NMW heuen y WM hewon. Desde Tyndale (T) y 1534, las protestantes persistirán en el singular.

El translema ó $\varphi \varepsilon \imath \lambda \eta ́ \mu \alpha \tau \alpha$ (opheilếmata) vale "deudas": debita, como dice la Vulgata. De nuevo y aunque la polisemia permita, en un sentido más teológico, pensar en "ofensas", por esa misma razón se podía haber propuesto "pecados" y carecería de ambigüedad: así lo hizo ELLC (sins). Además, inmediatamente, aparecerá peccata (Mat. 6:14), en función de sinónimo, junto a delicta. Como en el caso anterior, las versiones españolas, francesas e italianas -valdense incluida- concuerdan: deudas, dettes y debiti. Las alemanas se sirven de la útil polisemia de Schuld ("culpa", "deuda") y Schuldigern (“culpables", "deudores"). LG hace lo propio con scylda, mientras que A prefirió zyltas ("culpas", cf. inglés contemporáneo guilt). C1 da geltas ${ }^{9}, \mathrm{C} 2$ yeldinges $^{10}, \mathrm{NWM}$ mysdedes ("delitos") antepuesto a hom that trespas us ("los que yerran contra nosotros"), WM también prefiere mysdedus, T eligió treaspases ("yerros"), AV volverá a debts ("deudas") y el Anglican Book of Common Prayer de 1549 retomaría trespaces.

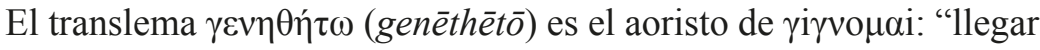
a ser", "hacerse", "nacer", "producirse", "realizarse", "manifestarse", "suceder", "resultar", o "cumplirse" y demás. El sucinto subjuntivo de presente, fiat, de la Vulgata se ha reproducido en castellano con el sentencioso, casi judicial hágase, frente a sia fatta y que (ta volonté) soit

\footnotetext{
${ }^{9}$ Obsérvese el típico cambio kéntico en el inglés medio de $y / \mathrm{I} /$ por $e$ /e/ tónicas.

${ }^{10}$ Con palatalización de oclusiva velar sonora ante vocal anterior, sonorización de oclusiva dental y nominalización sureña en -ing.
} 
faite ("sea hecha") del italiano y el francés. El alemán prefiere geschehe ("suceda"). LG y A eligieron sie y sy ("sea"). C1 gewurðe ("desarróllese", cf. alemán gewerde). C2 cambiará a be done ("sea hecha") y WM le seguirá. T prefirió be fulfilled ("sea cumplida") y AV vuelve a be done.

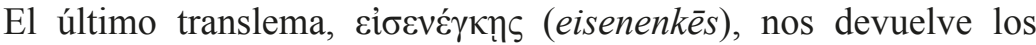
problemas teológicos. El latín inducas vale "conducir a", "llevar a", etimológicamente "inducir". CEI pone indurre, contra el valdense sporri "expongas". Muy al contrario, el francés y el español rechazan que Dios pueda tentar. El Dios del Antiguo Testamento sí que tienta, directamente o por mediación de Satanás, como en el Libro de Job y en Faust. El del Nuevo Testamento, no. Lo dice claramente Santiago en su epístola "católica": Nemo cum tentatur, dicat quoniam a Deo tentatur: Deus enim intentator malorum est: ipse autem neminem tentat. (Stg. 1: 13). Ambas culturas harán recaer, pues, la responsabilidad sobre el individuo: laissez pas succomber à y no nos dejes caer. Las versiones alemanas y todas las inglesas desde LG utilizan "conducir" -führe en alemán, lead en inglés- excepto ELLC, que parafrasea save us from the time of trial (literalmente: "guárdanos del tiempo/momento de la prueba").

\subsection{Conclusiones}

Hemos visto que las divergencias en Juan 1 no afectan tanto al tema, (uerbum) sino a la preposición (apud), cuya alteración puede protestar herejía. En el Cantar de los Cantares, la disensión abarca tanto el modificador (nigra) como la conjunción (sed). En el Padrenuestro no habrá acuerdo alguno: ni en los referentes físicos (caelis), ni en las opciones sinonímicas (debita), ni en la recreación de los verbos de devenir (fiat), ni en la posible identidad de acción entre el Dios del Antiguo y el de Nuevo Testamento (inducas).

El sosiego de Juliet ante las nuevas que le trae la nodriza pende de un adverbio monosílabo (RJ III, ii, 51-52), el Doctor de Los intereses creados (acto 2. ${ }^{\circ}$, cuadro 3. ${ }^{\circ}$, escena 9..) trastoca sentencias judiciales con una sola coma y, en Mutter Courage und ihre Kinder (Bild 4), la buhonera hace profesión de atea, prestidigitando coma en dos puntos. Nuestro corpus no llega a tanto, se queda en un estadio intermedio. Como presuponía la tesis de inicio, pasamos de la mayor fidelidad al TL1 del texto "informativo" a la mayor libertad de los textos "persuasi- 
vos". Pero, frente a lo que podríamos haber pensado (y salvo en el $\mathrm{Pa}$ dre Nuestro, porque no incluimos ninguno), los translemas gramaticales se revelarán como el quid, el nudo traductológico y desencadenarán las mayores discrepancias.

\section{Referencias bibliográficas}

\subsection{Referencias impresas}

Alonso Schökel, Luis. 1989. El Cantar de los Cantares. Estella: Verbo Divino. Delisle, Jean \& Woodsworth, Judith (ed.). 1995. Translators through history. Amsterdam / Philadelphia: John Benjamins Publishing Company/ UNESCO Publishing.

Dryden, John. 1975. Preface to Ovid's Epistles. En Steiner, T. R. English translation theory 1650-1800, 68.

El Nuevo Testamento de Nuestro Señor Jesucristo. 1983. Antigua Versión de Casiodoro de la Reina (1569). Revisada por Cipriano de Valera (1602). Otras revisiones 1862, 1909 y 1960. (RV). The New Testament of our Lord and Saviour Jesus Christ. Translated out of the original Greek and with the former translations diligently compared and revised commonly known as the Authorized (King James) Version. Los Gedeones Internacionales. (Edición de 1983). The Gideons International. (1983 edition). Copyright 1960. Sociedades Bíblicas en América Latina. Printed in U.S.A. National publishing Company (Cia. Publicadora Nacional). Philadelphia, Pennsylvania. (AV)

Horatius Flaccus, Quintus. 1967. Opera. Ars poetica. Edición a cargo de H. W. Harrod. Oxford: At the Clarendon Press.

Jerónimo, San. 1952. Cartas de San Jerónimo. Edición Bilingüe. I. Introducción, versión y notas por Daniel Ruiz Bueno. Madrid: Biblioteca de Autores Cristianos.

Kloepfer, Rolf. 1967. Die Theorie der literarischen Übersetzung. Romanischdeutscher Sprachbereich. München: Wilhelm Fink.

León, Fray Luis de. 1972. El cantar de los cantares. Barcelona: Ramón Sopena.

Moya, Virgilio. [2004] 2010. La selva de la traducción. Teorías traductológicas contemporáneas. Madrid: Cátedra.

Nida, Eugene. 1964. Toward a science of translating. With special reference to principles and procedures involved in Bible translating. Leiden: E.J. Brill. 
Nietzsche, Friedrich. 1969. "Zum Problem der Übersetzung” (extractado de "Die fröhliche Wissenschaft" y de "Jenseits vom Gut und Böse"). En Störig, Hans Joachim (ed.) Das Problem des Übersetzens..., 136-138.

Old English Homilies and Homiletic Treatises. (R. Morris, ed.). Oxford: Oxford University Press (E.E.T.S. Original Series) 1867 (reimpresión 1998).

Old English Version of the Gospels. (R. M. Liuzza, ed.). Oxford: Oxford University Press (E.E.T.S. Original Series) I. 1994 (reimpresión 2009), II. 2000.

Oldest English texts, The. (Henry Sweet, ed.). Oxford: Oxford University Press (E.E.T.S. Original Series) 1885 (reimpresión 1963).

Quintilianus, Marcus Fabius. 1971. Institutionis oratoriae libri XII. Compilado por L. Rademacher. Leipzig: Teubner Verlagsgesellschaft (Bibliotheca scriptorum graecorum et romanorum teubneriana).

Reiss, Katharina. 1971. Möglichkeiten und Grenzen der Übersetzungskritik. München: Max Hueber Verlag.

Reiss, Katharina \& Vermeer, Hans Josef. 1996. Fundamentos para una teoría funcional de la traducción. Madrid: Akal (Universitaria).

Roganova, Zoja E. 1971. Perevod s russkogo jazyka na nemeckiy. (Posobie na teorii perevoda dlja institutov i fakultetov inostrannyh jazykov) ("Traducción del ruso al alemán. (Manual de teoría de la traducción para los Institutos y Facultades de lenguas extranjeras)"). Moscú: Escuela Superior. En Vidal Claramonte, M. ${ }^{a}$ Carmen África. Traducción, manipulación, desconstrucción, 30-31.

Sagrada Biblia. 1973. (11. ${ }^{\mathrm{a}}$ ed.). Traducida de la Vulgata latina teniendo a la vista los textos originales por el P. José Miguel Petisco de la Compañía de Jesús. Dispuesta y publicada por el Ilmo. Sr. D. Félix Torres Amat. Madrid/Lima: Editorial Apostolado de la Prensa S.A.; y Zaragoza: Editorial Hechos y Dichos. (P-TA)

Sagrada Biblia. Versión directa de las lenguas originales. 1959. (9. a ed.). Eloíno Nácar Fuster (†) y Alberto Colunga, O.P. Prólogo del Excmo. y Rvdmo. Sr. Gaetano Cicognani, Nuncio de Su Santidad en España. Madrid: Biblioteca de Autores Cristianos. (N-C)

Santoyo, Julio César. 1986. A propósito del término TRANSLEMA. Actas del I congreso nacional de AESLA. Murcia, 14-17 de abril de 1983. Madrid: AESLA-Sgel, 255-265.

Stackelberg, Jürgen von. 1972. Literarische Rezeptionsformen. Übersetzung · Supplement - Parodie. Frankfurt am Main: Athenäum Verlag.

Steiner, George. 1975. After Babel: aspects of language and translation. Oxford: Oxford University Press. 
Steiner, T. R. 1975. English translation theory 1650-1800. (= Approaches to translation studies, 2). Assen/Amsterdam: Van Gorcum.

Störig, Hans Joachim (ed.). 1969. Das Problem des Übersetzens. StuttgartDarmstadt: Wissenschaftliche Buchgesellschaft.

The Holy Bible Containing the Old and New Testaments. 1980. Translated from the original tongues and with the former translations diligently compared and revised by His Majesty's special command. Appointed to be read in Churches. Trinitarian Bible Society. London: Eyre \& Spottiswoode Ltd. (AV)

Vidal Claramonte, M. ${ }^{a}$ Carmen África. 1995. Traducción, manipulación, desconstrucción. Salamanca: Ediciones del Colegio de España (Biblioteca Filológica).

\subsection{Referencias en línea}

http://simplebibletruths.net/70-John-1-1-Truths.htm

https://www.biblegateway.com/

http://www.fourmilab.ch/etexts/www/Vulgate/

http://www.ellopos.net/elpenor/greek-texts/septuagint/chapter.asp?book=28

http://www.hs-augsburg.de/ harsch/Chronologia/Lspost04/Hieronymus/hie v000.html

http://www.ling.upenn.edu/ kurisuto/germanic/oe_bosworthtoller_about.html

\section{Otras ediciones bíblicas consultadas, impresas y en línea}

A Aelfric. Homily on the Lord's Prayer, aprox. 1000. Cambridge University Library MS Gg 3. (o)

AMP Amplified Bible (1987). (z)

AMPC Amplified Bible Classic Edition. (z)

ASV American Standard Version (1900). (h)

BDS La Bible du semeur (1992). (h)

C1 Canterbury. Homily on the Lord's Prayer de Alfric, copiada aprox. 1200. Bodleian. Library. MS Hatton 38. (o)

C2 Canterbury. 1340. British Library. MS Arundel 57. (o)

CEI Conferenza episcopale italiana (2008). (o)

CEV Contemporary English Version or Biblefor Today's Family(1995).(z)

DHH Dios habla hoy (1979). (z)

ELLC English Language Liturgical Consultation (1988). (z)

G Geneva Bible (1599). (h)

GNT Good News Translation (1966). (z)

L Luther Bibel (1545). (h) 
LBG La Bibbia della Gioia (2008). (o)

LG Lindisfarne Gospels. Glosa a los Evangelios de Lindisfarne, aprox. 950. British Library MS Cotton Nero D.IV (o)

LND La Nuova Diodati valdense (1991). (h)

LSG Louis Segond (1880). (h)

NABRE New American Bible Revised Edition (2011). (o)

NEG Nouvelle edition de Genève (1979). (h)

NR Nuova Riveduta (1994 y 2006). (h)

NTV Nueva traducción viviente (2010). (h)

NWM North-West Midland. aprox. 1375, en Lay Folk's Mass Book. British Museum. MS Royal 17 B xvii. Vid. también la edición a cargo de F. T. Simmons. Oxford: Oxford University Press (E.E.T.S. Original Series) 1897 (reimpresión 1968) (o)

S Schlachter (1951 y 2000). (h)

T Tyndale (1534). (h).

TLA Traducción en lenguaje actual (2003). (h)

TLB The Living Bible (1971). (h)

W Wycliff(1382-1395). (h)

WE Worldwide English (1969). (h)

WET The Wuest expanded translation (1956). (z)

WM West Midland. aprox. 1450. Cambridge. Gonville and Caius College. MS 84.162. (o)

Símbolos: (o) católica, (h) protestante, (z) adscripción no fijada, no clara o "interconfesional".

NB: Agradezco al Dr. D. Miguel Fuster Márquez la erudición de las versiones del Padrenuestro entresacadas de la historia de la lengua inglesa. 
